172 CARDIAC AND EXTRACARDIAC AMYLOIDOSIS IN V122I ATTR

Aisling Carr, ${ }^{1}$ Z Jaunmuktane, ${ }^{1}$ Healy Pelayo, ${ }^{1}$ D Hutt, ${ }^{2}$ S Brandner Holton, ${ }^{1}$ J Blake, ${ }^{1}$ CJ Whelan, ${ }^{2}$ AD Wechalekar, ${ }^{2}$ JD Gilmore, ${ }^{2}$ PN Hawkins, ${ }^{2}$ MM Reilly'. ' $U C L ;{ }^{2} N A C$

10.1136/jnnp-2014-309236.172

Introduction Hereditary transthyretin-related amyloidosis (ATTR) is a genetically heterogenous disease which may present with a predominant peripheral and autonomic neuropathy, a cardiac myopathy, or with both neurological and cardiac involvement. The V122I variant has a frequency of 3-4\% in African Americans and causes 11\% of cardiomyopathy in this population. Isolated cardiomyopathy is the typical presentation. Methods Here we present the clinical, electrophysiological, nuclear imaging and histological findings of an unusual case of V122I ATTR.

Results A 64 year old Jamaican man presented with cardiac failure. Cardiac MR revealed an infiltrative cardiomyopathy; abdominal fat aspirate confirmed the presence of amyloid and a homozygous V122I mutation in the transthyretin gene were identified. He also described positive sensory symptoms in his feet and general, non-specific weakness; NCS showed length-dependant axonal neuropathy and EMG demonstrated some myopathic features. Amyloid deposits were seen on sural nerve and vastus lateralis biopsies. The patient is being treated with diflunasil, an oral agent with in vitro evidence for TTR stabilization. 


\section{ABN Abstracts}

Conclusion Symptomatic neuropathy or myopathy with morphological confirmation of tissue amyloid deposition has not been previously described with this mutation. The presence of extracardiac amyloidosis impacted on diagnostic and treatment options in this case. 